\title{
Ligament Reconstruction and Tendon Interposition Using Flexor Carpi Radialis Tendon for the Treatment of Basilar Thumb Arthritis: A Case Report
}

\author{
Andini Febriana $^{1}$, Agus Eka Wiradiputra $^{2}$ \\ ${ }^{1}$ Intern of Orthopaedics and Traumatology Department, Bhayangkara Denpasar Hospital \\ ${ }^{2}$ Consultant of Orthopaedics and Traumatology Department, Bhayangkara Denpasar Hospital \\ Corresponding Author: Andini Febriana
}

\begin{abstract}
Introduction: Basilar thumb arthritis is most common arthritis affected about $10 \%$ of middle aged women and one third of postmenopausal women had this condition. The disease is often not shown significant symptom, when become a symptomatic can provide 50\% loss of function. Conservative treatment with mild arthritis usually had a good respond. Surgery is indicated in patients who failed to conservative treatment. Ligament reconstruction and tendon interposition is the surgical approach commonly used to treat basal joint arthritis.
\end{abstract}

Case Presentation: A 61-year-old woman complained swelling and also pain at the base of the left thumb more than 1 year and unable to do a daily activity and getting worse, also complained weakness grip. Plain radiograph of hand showed the trapezial space height about $10 \mathrm{~mm}$ and diagnosed with basilar thumb arthritis of the left hand Eaton stage III with DASH score pre operatively is 60.8 . The patient done with LRTI procedure and used thumb spica cast for immobilized 4 weeks. The DASH Score Post operatively is 40.7 and after 6 months the score is improve to 19.2.

Conclusion: Basal Thumb Arthritis is a common condition affecting postmenopausal women with a deformity consisting of a MCP hyperextension deformity accompanied by swelling, tenderness and crepitus. Eaton Classification used to staging of basal thumb arthritis and help to decide the treatment. LRTI is one approach to treat patients with CMC arthritis who has a high level of satisfaction and objectivity in measuring function, including restoring of the strength and ability of the affected thumb.

Keyword: Basilar, Thumb, Joint, Arthritis, Ligament reconstruction, tendon interposition

\section{INTRODUCTION}

Basal joint osteoarthritis is the problem commonly affected to the thumb. The second most common osteoarthritis (OA) in the hand other than the distal interphalangeal (IP) joint of the index finger. ${ }^{1}$ A common condition that affects up to $10 \%$ of middle-aged women and about one-third of postmenopausal women. ${ }^{2,3}$ Although the prevalence of this disease is high, it is often not showing significant clinical symptom, and most of the patients not seeking for the treatment. When this condition become symptomatic, decreasing function of the thumb can provide damage up to $50 \%$ of the upper extremity. ${ }^{4,5}$ Many literature and researches suggest the etiologies of basilar thumb arthritis, including genetics problem, ligament laxity, post traumatic arthritis and overuse of the thumb. A progressive of the arthritis develops gradually in a characteristic pattern of wear and tear, sometimes at the end resulted in pantrapezial arthritis. ${ }^{1,6}$ The conservative treatment usually has a good result especially for mild arthritis patients. Surgery is indicated to the patient with 
severe arthritis and patients who not showing good respond to conservative treatment. ${ }^{2,4}$ Many surgical approaches such as tendon interposition, ligament reconstruction, distraction hematoma (HDA) arthroplasty, carpometacarpal arthroplasty or arthrodesis, has been described but no one that shows its superiority over the others. ${ }^{7}$ The approach that frequently used to treat the basillar thumb arthritis is ligament reconstruction and tendon interposition (LRTI). In 1986 Burton and Pellegrini was the first person who introduced this technique. The concept of this procedure was a combination of the volar ligament reconstruction as described by Eaton and Littler and interposition arthroplasty by Froimson. ${ }^{1,2,7}$ This article was reported the case of basal joint arthritis who treated with LRTI, and the patient was evaluated the functional outcome with DASH Score.

\section{CASE PRESENTATION}

A 61 year old woman with a chief complaint of swelling and pain around the left thumb especially at the base regions for more than 1 year, she was unable to use her hand for daily activity and getting more worse in last 3 months, she also complained about having weakness when grip something. The plain radiograph of the hand

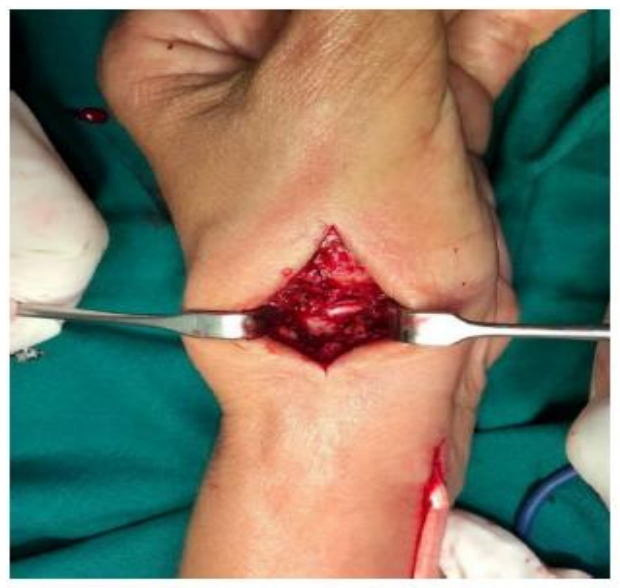

Figure 2: Removing trapezium

The aim of this step was to make more space to move the thumb, so that the arthritic bone surfaces are not rubbing together causing pain. After done removed showing the trapezial height to be $10 \mathrm{~mm}$ and the arthritis at carpometacarpal joint of the left thumb, as shown in figure 1. The left basilar thumb arthritis Eaton stage III was diagnosed to the patient with DASH score pre operatively is 60.8 .

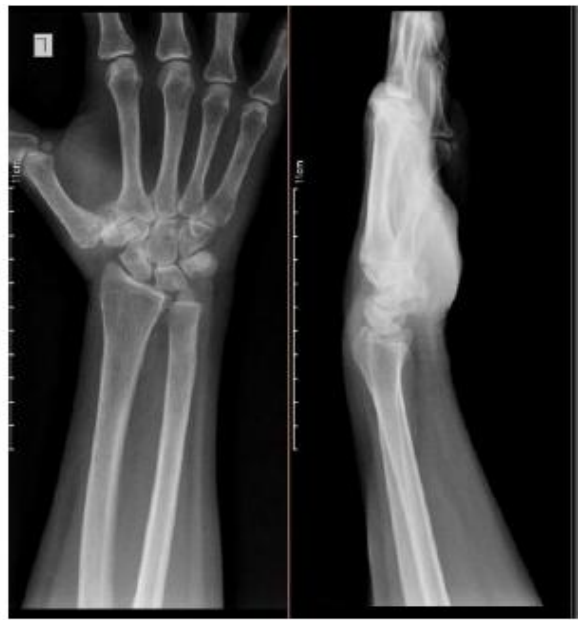

Figure 1: Anteroposterior and Lateral Radiograph shows the trapezial height space and the arthritis of carpometacarpal (CMC) left thumb.

On this patient, we performed general anesthesia with hand on supine position and following incision at the dorsal thumb metacarpal bone with longitudinal incision. After the trapezium bone was exposed, and the superficial branch of the radial nerve was protected, we removing the trapezium, at the base of the thumb (figure 2).

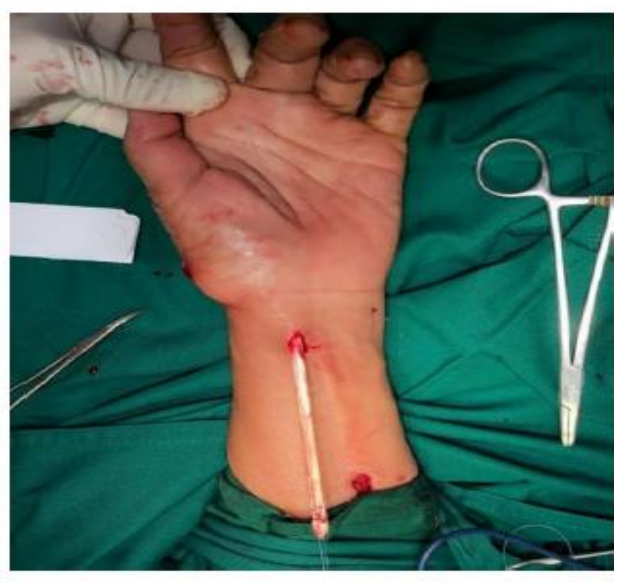

Figure 3: Harvesting flexor carpii radialis

trapezium, we make a hole with drilling from the dorsal base to the articular surface, following with a short transverse incisions at the distally and proximal of forearm, after 
that the FCR was split longitudinally into two halves, and passed half of it through the hole and tied it to another part and we sutured them, we harvested around 10 to12 $\mathrm{cm}$ of the flexor carpi radialis (FCR) tendon. This suture is purposed to protect the FCR tendon (figure 3 ).
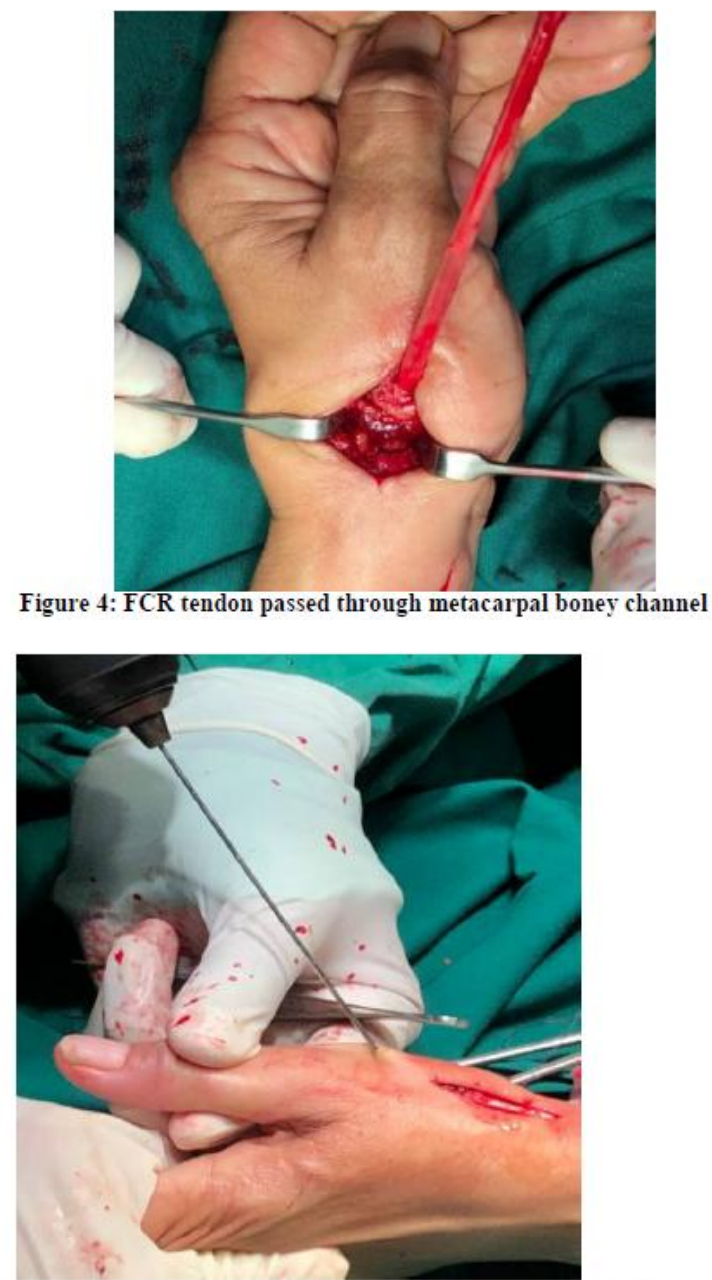

Figure 6: Using $\mathbf{K}$-wire to provide temporary mechanical suspension

We sutured the tendon ball into the volar capsule and also to the ligament. After that we closed the capsule, the aim is to prevent it from escaping. We also using the Kirschner wire to provide temporary mechanical suspension, it was passed from first to second metacarpal (figure 6).

This procedure was ended with the closure of the soft tissue with absorbable sutures and the skin was closure by nylon sutures. After completing all the surgical procedures, we immobilized the thumb with the short arm spica cast for 4 weeks. The DASH score was measured post operatively and the result is 40.7. Follow up after 3
After FCR tendon was tied, it was passed through the metacarpal base bony channel (figure 4), and we make the tendon into a ball with rolled up the tendon, then put into the space where trapezium was removed. (figure 5)

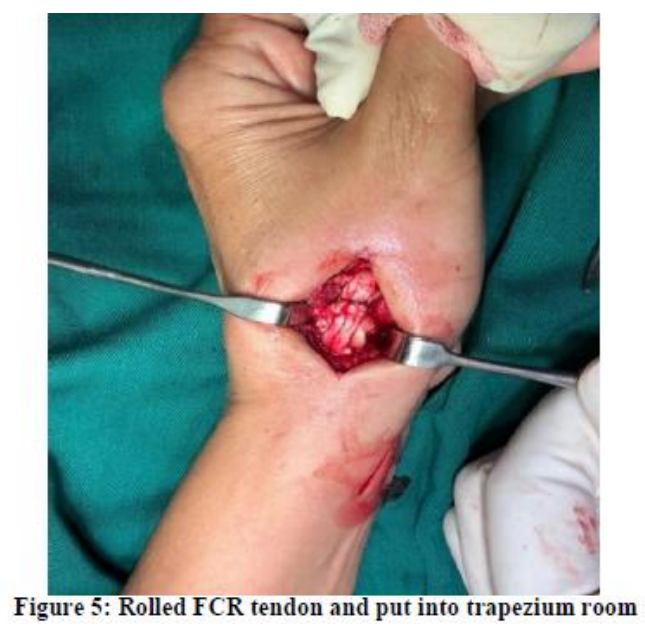

months post operatively, DASH score was 23.3 and after follow up 6 months the score was improved into 19.2.

\section{DISCUSSION}

CMC joint arthritis is a common condition which affects most women over the age of 45, and about one-third of postmenopausal women. ${ }^{1,5,8}$ Patients usually describe pain or activity-related tenderness at the thumb's base, Followed by pinching and grasping difficulty. The early stages of arthritis may show normal examination of the thumb. Nevertheless, at a later stage, the deformity of the metacarpals mimicking consist of an adduction position followed by a compensator hyperextension MCP deformity. The examination might show swelling, tenderness, and crepitation ${ }^{1,9,10}$ Same like our case the patient was $61^{\text {st }}$ year old women in postmenopausal period complains sore and tenderness also swollen at the base of her left thumb and also complaining about weakness when grip something. Weiss et al, said conventional radiographs in order to evaluation the basal thumb joint arthritis must contain anteroposterior and lateral, also oblique views of wrist or hand. Arthritis of the 
thumb is most common, which described with the Eaton-Littler classification, in 1973 and was modified by Eaton and Glickel in 1987 (Figure 7). ${ }^{1,4}$ The classification of this patients is The Eaton Classification stage III based on the radiograph shown the height of the trapezial space widening $10 \mathrm{~mm}$.

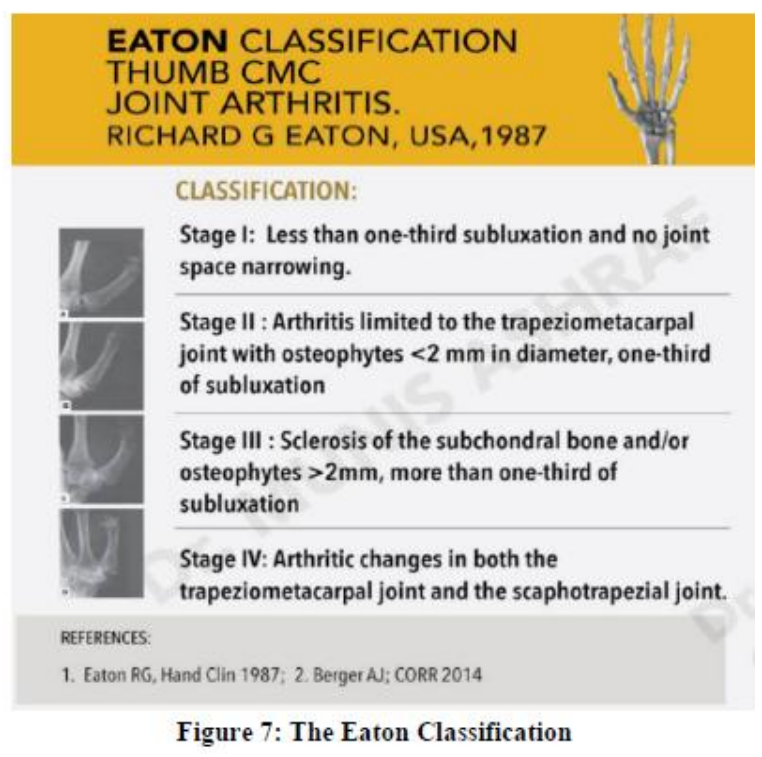

The Eaton Stages System is used to decide the approach in treatment of basal thumb arthritis. Initial treatment with conservative therapy in the form of NSAIDs and splinting with short opponens splint shows beneficial in stage I arthritis and initial Stage II. The trapezial excision is performed while there is an increase in joint wear, it performed whole or partial excision, with or without interpositioning material (autograft tendon or allograft tendon), and with or without ligament reconstruction. This approach is required in the management of patients with stage III and IV of basal thumb arthritis. Nowadays, surgeons have using back to only the trapezial excision which described as hematoma arthroplasty and has profitable short-term outcomes, despite loss of height at trapezoid space with subsequent impingement of the scaphoid is a concerned long-term consequence that can affect longterm surgical outcome. ${ }^{11,12}$

Prevention of disturbance, the modified of the simple trapeziectomy can be an alternatives. Includes interpose of alloplastic or autogenous tissue between metacarpal's base and carpometacarpal, rebuild the supporting ligaments with many type of tendons, and CMC arthrodesis. Our case is CMC arthritis stage III with weakness when grip, the operative treatment is choose to treat the patient. ${ }^{8,12}$

Huang et all, said on his research that many other surgical treatments, such as prosthesis arthroplasty, trapeziectomy with hematoma distraction arthroplasty or tendon interposition, are good solutions for basal joint arthritis for in order to preserve the scaphotrapezial joint. While degenerative deformity in the joint surface of the scaphotrapezial, the functional outcomes would be tolerate. In situations where concern about scaphotrapezial joint problems, LRTI can be an option, this procedure has a good responds, without much concern about the first metacarpal proximal migration in the future. ${ }^{2}$

Many literatures mention that LRTI has felicitous assurance and great objective of function, includes strength and dexterity reversion to the gravely affected thumb. Brinkley et all compared LRTI with HDA and the scored mutually on QuickDASH. Many declared great improve of pain, tip pinch, lateral pinch and average grip, were mutual in those two groups, neither one achieved significant number, on every levels of subjective and objective measurements, either the purpose to achieved pain-free and stable motion of the thumb, this also mentioned at Kuhns et all research too. However even the result has similarity on QuickDASH score, Brinkley et all mention that LRTI still shown improvement in strength, while HAD has possibility for escalated proximal migration which causes a weaker thumb and pain from the support on the distal scaphoid. ${ }^{4}$

Abbas et al, said the LRTI arthroplasty produces great pain relief. Build based on observation of Quick DASH scores, the outcomes have been constantly supported in most cases with recovery of normal anatomy to produce stability and functional of thumb. ${ }^{5}$ 
Tomaino et al, report ligament reconstruction-tendon interposition arthroplasty produces great pain relief and a necessary improvement in strength, based on long-term follow-up study, with follow up until nine years (with range up until 8 until 11 years), $91 \%$ of participants encounter complete relief of pain and were satisfied with the results of the operation, this result also similar to $87 \%$ satisfaction rate of Vinycomb et all study that analyze 15 cases of trapeziectomy with LRTI follow up was range 10.5-17.5 yrs, a decent results was a participant did not need restoration surgery, and satisfied with the result of their surgery and did not encounter rest pain. ${ }^{13}$

Based on many literature discuss about the excellent of LRTI to become a operative treatment approach to treat CMC arthritis, we choose LRTI procedure to treat our patient. The DASH Score is quantitative scoring that we used to measurement the patient's functional outcome. Disabilities of the Arm, Shoulder, and Hand (DASH) score was conducted to be a standardized evaluation of the effect on function of many types of musculoskeletal disease and defects in the upper extremity, DASH consist of 30 items questionnaire, which the answers are served as 5-point Likert scales. Which score range from 0 (no disability) to 100 (most disability). Primarily published in 1996 in the American Journal of Industrial Medicine, there was an initiative collaboration between the American Academy of Orthopaedic Surgeons, the Council of Musculoskeletal Specialty Societies, and the Institute for Work and Health. The score was conducted as to be useful in patients with musculoskeletal disorder of the upper limb. ${ }^{14}$ Pre operatively DASH Score of our patient is 60.8 and was improve when we measure 3 months post operative 40.7 and after follow up 6 months the score is 19.2

\section{CONCLUSION}

Basal Thumb Arthritis is common problem affecting postmenopausal women mostly over 45 years of age, with the symptom are deformity consisted of an added position accompanied by a compensatory MCP hyperextension deformity. Physical examination might express swelling, tenderness, and crepitation. Eaton Classification was used to staging of CMC arthritis and help us to decide the treatment to the patient. LRTI is one of many approaches to treat the patient with CMC arthritis that has a high satisfaction rate and good objective value of function, including return of strength and dexterity in the severely affected thumb.

Funding: The authors received no financial support for the research, authorship, and/or publication of this article.

Disclosure of Interest: The authors declare that they have no conflicts of interest concerning this article.

\section{Acknowledgement: None}

\section{Conflict of Interest: None}

\section{REFERENCES}

1. Weiss APC, Goodman AD. Thumb Basal Joint Arthritis. J Am Acad Orthop Surg 2018; 26: 562-571.

2. Huang YC, Huang HK, Liu YA, et al. Longterm results of modified ligament reconstruction and tendon interposition for thumb basal joint arthritis. J Chinese Med Assoc 2019; 82: 655-658.

3. Dias R, Chandrasenan J, Rajaratnam V, et al. Basal thumb arthritis. Postgrad Med J 2007; 83: 40-43.

4. Sandvall BK, Cameron TE, Netscher DT, et al. Basal joint osteoarthritis of the thumb: Ligament reconstruction and tendon interposition versus hematoma distraction arthroplasty. J Hand Surg Am 2010; 35: 1968-1975.

5. Abbas K, Hashmi P, Raza H. Short term results of ligament reconstruction and tendon interposition resection arthroplasty for basal joint arthritis. J Pak Med Assoc 2012; 62: 398401.

6. Shuler MS, Trumble TE. Thumb.

7. Rhee PC, Paul A, Carlsen B, et al. Outcomes of Surgical Management for Thumb Basilar Arthritis in Patients 55 Years 
Andini Febriana et.al. Ligament reconstruction and tendon interposition using flexor carpi radialis tendon for the treatment of basilar thumb arthritis: a case report

of Age and Younger. Hand 2019; 14: 641645.

8. Gangopadhyay S, McKenna H, Burke FD, et al. Five-to18-year follow-up for treatment of trapeziometacarpal osteoarthritis: A prospective comparison of excision, tendon interposition, and ligament reconstruction and tendon interposition. J Hand Surg Am 2012; 37: 411-417.

9. Burton RI, Pellegrini VD. Surgical management of basal Joint arthritis of the thumb. Part II. Ligament reconstruction with tendon interposition arthroplast. J Hand Surg Am 1986; 11: 324-332.

10. Elfar JC, Burton RI. Ligament Reconstruction and Tendon Interposition for Thumb Basal Arthritis. Hand Clin 2013; 29 : $15-25$.

11. Kriegs-Au G, Petje G, Fojtl E, et al. Ligament reconstruction with or without tendon interposition to treat primary thumb carpometacarpal osteoarthritis. J Bone Jt Surg - Ser A 2005; 87: 78-85.

12. Gong HS, Chung MS, Oh $\mathrm{JH}$, et al. Ligament Reconstruction and Tendon Interposition for Advanced Posttraumatic Arthritis of the Proximal Interphalangeal
Joint: 3 Case Reports. J Hand Surg Am 2008; 33: 1573-1578.

13. Wang T, Zhao G, Rui Y, et al. Outcomes of modified trapeziectomy with ligament reconstruction tendon interposition for the treatment of advanced thumb carpometacarpal arthritis: Two-year followup. Medicine (Baltimore). 2018 Mar;97(13): e0235. doi: 10.1097/MD. 0000000000010235.

14. Hudak PL, Amadio PC, Bombardier C. Development of an upper extremity outcome measure: The DASH (disabilities of the arm, shoulder, and head). Am J Ind Med. Epub ahead of print 1996. DOI: 10.1002/(SICI) 10970274(199606)29:6<602: :AID-AJIM4>3.0.CO;2-L.

How to cite this article: Febriana A, Agus Eka Wiradiputra. Ligament reconstruction and tendon interposition using flexor carpi radialis tendon for the treatment of basilar thumb arthritis: a case report. International Journal of Science \& Healthcare Research. 2021; 6(3): 267-272. DOI: https://doi.org/10.52403/ijshr. 20210746 